\title{
Effects of Light Quality on Growth, Total Gypenosides Accumulation and Photosynthesis in Gynostemma pentaphyllum
}

creative commons

Botanical Sciences 95 (2): 235-243, 2017

DOI: 10.17129/botsci.667

Copyright: (C) 2017 Peng et al. This is an open access article distributed under the terms of the Creative Commons Attribution License, which permits unrestricted use, distribution, and reproduction in any medium, provided the original author and source are credited.
Xiaolie Peng, Ting Wang, Xingyun Li, Shibiao Liu*

\section{Abstract}

Background: Gynostemma pentaphyllum is a kind of shade-tolerant plants. It can synthesize and accumulate gypenosides in large amounts. The gypenosides are a valuable medicine that against hypertension, hyperlipemia, obesity, neurological disorder, inflammation, immune-modulation, diabetes and tumours.

Hypotheses: Light quality was proved to affect the growth and gypenosides accumulation obviously in $G$. pentaphyllum, and it affects the photosynthesis certainly. So, some relationships may exist among these parameters.

Study site and dates: Experiments were carried out in the late May of 2014 at Jishou University, Hunan province, China.

Methods: Measurements of growth features and photosynthesis as well as determination of total gypenosides were conducted in seedlings that planted under different light qualities, including white, red, blue and green light emitted by LED lamps.

Results: Light quality had remarkable effects on plant growth, total gypenosides accumulation and photosynthesis. White light had significant effects on increases in biomass, stem diameter and newly sprouted leaves than homogeneous light did. Compared with blue and green light, white light presented a higher content of total gypenosides which was closest to that of red light, suggesting the effect of white light on gypenosides accumulation was involved in red light. The net photosynthetic rate under red light was higher than those under blue or green lights, but obviously lower than that under white light.

Conclusions: It is suggested that the influence of light quality on growth and the accumulation of total gypenosides was associated with photosynthesis efficiency.

Key words: Gynostemma pentaphyllum, light quality, growth, total gypenosides, phototsynthesis
College of Biology and Environmental Science, Jishou University, Jishou, Hunan 416000, China

* Corresponding author: liushibiao_1@126.com 
ynostemma Blume (Cucurbitaceae) is a genus of climbers comprising about 17 species and 2 varieties located throughout Asian. The largest species diversity is found in China. The most prevalent species is G. pentaphyllum (Thunb.) Makino (namely Jiaogulan in Chinese), a perennial creeping herb. The species is dispersed throughout the south-western region of China as well as some areas in India, Nepal, Bangladesh, Sri Lanka, Laos, Myanmar, Vietnam, Korea and Japan (Blumert \& Liu 1999). Due to its capability to synthesize and accumulate gypenosides which own the same dammarane type structure as ginsenosides, the G.pentaphyllum has been honored as "Southern Ginseng" in China, and therefore, is becoming an important source of medicinal plants. Apart from the traditional use in defense against hypertension, hyperlipemia, obesity and neurological disorders, the gypenosides have been used for their antiinflammatory properties (Lüthje et al. 2015), immune-modulatory functions (Im et al. 2012), antidiabetic and antitumour effects (Huyen et al. 2012, Yan et al. 2014). It was predicted that supplement products of $G$. pentaphyllum would be likely equivalent to ginsenosides (Chang et al. 2005). Currently, the G. pentaphyllum is widely grown in the areas of South China to provide different beneficial supplements which are being used as healthy tea, tablet, granule, and vegetarian capsule, and food addictive. The Jiaogulan tea become increasingly popular as a nutritional supplement, even in Europe and North America, together with other edible plants from traditional Chinese medicine, in the management of lifestyle-related health problems (Liu et al. 2015).

As a shade-tolerant plant, the morphogenesis, photosynthetic physiology and accumulation of secondary metabolities of Gynostemma pentaphyllum are influenced by both light intensity and light quality or light color. It is well known that the morphology and structure are directly affected by light intensity. For example, leaves under full sunlight have features of sun-leaves, but those under shading appear shade-leave features (Guo et al. 2008, Liu \& Hu 2004). About 40-85\% relative illumination intensity was beneficial to the growth of G. pentaphyllum, especially that of $70 \%$ of full sunlight (Deng et al. 2000). As for light quality, Wei et al. (2003) reported that blue light and red/orange light in forest gaps were more effective with respect to the net photosynthetic rate, total dry matter weight and total saponin content. Available evidence suggest red and orange lights were more effective than blue and purple lights in promoting the accumulation of total gypenosides in G. pentaphyllum (Liu et al. 2011, Li et al. 2011).

Photosynthesis is considered to be a key conversion of sunlight into growth and the accumulation of gypenosieds in G. pentaphyllum. Although several studies have focused on understanding the photosynthesis characteristics of this plant, little is known about the relationship between light quality and gypenosides accumulation associated with the photosynthesis and growth in this plant (Huang et al. 2004, Liu \& Hu 2004). Recently, light-emitting diodes (LEDs) have been developed as an alternative light source for plants because of their specific wavelength, narrow bandwidth, small mass, solid state construction, long life as well as minimum heating. Therefore, it is more convenient and accurate to investigate the effects of light quality on plant growth, development, photosynthesis, primary and secondary metabolism by using LEDs (Morrow 2008, Darko et al.2014). To develop effective cultivation techniques of the plant, effects of light quality on growth, accumulation of total gypenosides and photosynthesis in G. pentaphyllum were investigated in this study.

\section{Materials and methods}

Plant materials and treatments. The tissue cultured seedlings of Gynostemma pentaphyllum were transplanted into pots of $20 \mathrm{~cm}$ in diameter and $15 \mathrm{~cm}$ in depth. The pots then were filled with a mixture of peat and vermiculite $(1: 1, v / v)$, watered every two days with Hoagland solution in a greenhouse. 20 days later, 120 uniform seedlings with three fully expanded leaves were selected and subdivided into 4 groups. Each group contained 30 seedlings. Experiments were carried out in the late May of 2014 at Jishou University, Hunan province, China. Different LED lamps that emit red light $(625 \pm 20 \mathrm{~nm})$, green light $(525 \pm 20 \mathrm{~nm})$, blue light $(470 \pm 20 \mathrm{~nm})$ and white light (control check) were used respectively. Four boxes were made of wood strips with 1 $\mathrm{cm}$ interval. 400 lamps of LEDs were installed in a plate of $1.2 \times 1.2 \mathrm{~m}$ of the each box. A dark cloth was used to wrap the box. 30 seedlings were placed in each box and exposed to different 
color lights under a $12 \mathrm{~h}$ photoperiod with $100 \mu \mathrm{mol} \cdot \mathrm{m}^{-2} \cdot \mathrm{s}^{-1}$ light intensity (by adjusting the distance between plant apex and light source) for 60 days before sampling.

Chemicals. Standard sample of total gypenosides $(99 \%)$ were obtained from the Chinese National Institute for the Control of Pharmaceutical \& Biological Products (Beijing, China) and used as standards in the quantification of total gypenosides. Other reagents in this study were analytical grade and purchased from Sangon Biotech (Shanghai, China) Co., Ltd.

Measurement of photosynthesis. The fifth newly sprouted and fully expanded leaves from 3 individual plants were chosen to measure net photosynthetic rate $(\mathrm{Pn})$, stomatal conductance (Cond), transpiration rate $(\mathrm{T})$ and intercellular $\mathrm{CO}_{2}$ concentration $(\mathrm{Ci})$, using a portable photosynthesis system (LICOR-6400 ${ }^{\circledR}$ LI-COR Inc., USA) from 8:00 am to 18:00 pm of 3 continuous fine days in the middle August of 2014 at 2 hour intervals for each treatment.

Measurement of growth. After the photosynthesis measurement, the boxes were uncovered. The third newly sprouted leaf was chosen to measure the leaf area with a leaf area scanner. A digital vernier caliper was used to measure the petious length of the fifth newly spouted leaf and the stem diameter of the internode between the forth and fifth newly spouted leaves. The total length of the newly grown stem was measured with a measure tape. The newly sprouted leaves were counted. After the measurements, the new sprouted leaves and stems were cut off and dried in an oven at $60{ }^{\circ} \mathrm{C}$ for 48 hours until constant weight to determine the biomass. An ANOVA multivariate analysis of variance at $5 \%$ level was done on the growth parameters using SPSS software. All data was expressed as mean $\pm \mathrm{SD}$ with statistical significance at $P \leq 0.05$.

Determination of total gypenosides. The dried samples were ground in a mortar and the powders were collected by sifting them through a 10 mesh sift, stored in a vacuum container at room temperature until determination of total gypenosides. The total gypenosides in $1.0 \mathrm{~g}$ of powder was extracted with $10 \mathrm{~mL}$ of $75 \%$ ethanol for 10 hours at room temperature, followed by a $40 \mathrm{~min}$ water bath at $60{ }^{\circ} \mathrm{C}$ in ultrasonic instrument. The extractant was collected and centrifuged for 5 $\mathrm{min}$ at 5,000 rpm at room temperature. The supernatant was then added into a rotatory evaporator to remove the ethanol solvent by distillation under reduced pressure at $60{ }^{\circ} \mathrm{C}$ until $15-20 \%$ of ethanol was left. The solution was stored at $4{ }^{\circ} \mathrm{C}$ for 12 hours, followed by centrifugation at $5,000 \mathrm{r} / \mathrm{min}$ for $5 \mathrm{~min}$. Vacuum evaporation was used again to totally remove the ethanol from the solution. The residual solution was extracted with $\mathrm{H}_{2} \mathrm{O}$-saturated butanol for 4 times. The water layer was discharged and the butanol phase was collected and vaccumly evaporated. Five volume of ethyl ether was added to the concentrated residue and stored for 12 hours. The stored residue was centrifuged at $5,000 \mathrm{r} / \mathrm{min}$ for $5 \mathrm{~min}$. The supernatant was discharged. The precipitation was collected and dried at $60^{\circ} \mathrm{C}$. What remained was a light yellowish powder, the crude total gypenosides. The powder was dissolved with methanol in a constant volume of $50 \mathrm{~mL}$ to determine the concentration of the total gypenosides.

Were dissolved $10 \mathrm{mg}$ of gypenoside standard with methanol into $10 \mathrm{~mL}$, shaken up and diluted into $1 \mathrm{mg} / \mathrm{mL}$ standard solution. $0,20,40,60,80,100$ and $120 \mu \mathrm{L}$ of the solution gradients were prepared. $0.2 \mathrm{~mL}$ of freshly prepared $5 \%$ vanillin in acetic solution and $0.8 \mathrm{~mL}$ of perchloric acid were then added into each of the gradient solutions and mixed. The mixture was warmed up in a water bath at $60{ }^{\circ} \mathrm{C}$ for $15 \mathrm{~min}$. After cooling down to room temperature, $5 \mathrm{~mL}$ of glacial acetic acid was added. The absorbance of the standard solutions was measured colorimetrically at $550 \mathrm{~nm}$ (723A - Spectrophotometer, Shanghai Precision Instruments Co., Ltd. China). High linearity $\left(r^{2}>0.99\right)$ was gained for standard curve. The quantification of total gypenosides of each sample solution was performed on the basis of absorbance with comparison to the standard curve formula.

\section{Results}

Effects of different light quality on growth. The growth data of seedlings that were grown under different light qualities was listed in Table 1. Among the 4 kinds of spectrums, there was no sig- 
Table 1. Growth parameters of Gynostemma pentaphyllum seedlings grown under different light quality

\begin{tabular}{|c|c|c|c|c|c|c|}
\hline Light quality & $\begin{array}{l}\text { Leaf area } \\
\qquad\left(\mathrm{cm}^{2}\right)\end{array}$ & $\begin{array}{c}\text { Petious } \\
\text { length }(\mathrm{cm})\end{array}$ & $\begin{array}{c}\text { Stem } \\
\text { length }(\mathrm{cm})\end{array}$ & $\begin{array}{l}\text { Stem diameter } \\
(\mathrm{mm})\end{array}$ & $\begin{array}{l}\text { Number of } \\
\text { new leaf }\end{array}$ & $\begin{array}{l}\text { Dry weight } \\
\text { (g) }\end{array}$ \\
\hline White light(CK) & $16.14 \mathbf{a} \pm 3.32$ & $3.04 \mathbf{b} \pm 0.86$ & $58.02 \mathbf{a} \pm 29.21$ & $0.96 \mathbf{a} \pm 0.12$ & $19 \mathbf{a} \pm 7.76$ & $4.22 \mathbf{a} \pm 0.11$ \\
\hline Red light & $13.30 \mathbf{a} \pm 4.91$ & $3.80 \mathbf{a b} \pm 0.73$ & $58.44 \mathbf{a} \pm 33.03$ & $0.86 \mathbf{b} \pm 0.10$ & $14 \mathbf{b} \pm 6.22$ & $3.98 \mathbf{a} \pm 0.08$ \\
\hline Blue light & $15.26 \mathbf{a} \pm 3.93$ & $4.50 \mathbf{a} \pm 0.94$ & $37.27 b \pm 20.54$ & $0.89 \mathbf{b} \pm 0.17$ & $11 \mathrm{c} \pm 4.62$ & $2.67 \mathbf{b} \pm 0.05$ \\
\hline Green light & $14.06 \mathbf{a} \pm 4.77$ & $3.80 \mathbf{a b} \pm 0.75$ & $36.09 b \pm 17.40$ & $0.86 \mathbf{b} \pm 0.11$ & $9 c \pm 4.05$ & $2.27 \mathbf{b} \pm 0.05$ \\
\hline
\end{tabular}

nificant difference in leaf area although the leaf area under white light was relatively large. The petiole length under blue light was markedly longer than the control, and slightly larger than that under red and green lights. The stem length under white and red lights was significantly longer than that under green and blue lights, while the stem diameter under white light was observably larger than that under different homogeneous lights. Moreover, seedlings produced more newly sprouted leaves and the highest dry weight under white light than that from different homogeneous lights. On the contrary, seedlings under green light produced the least number of new leaves and dry weight. The number of new leaves and dry weight under red light were remarkably greater than those under blue and green lights, but were less than those under white light. The dry weight of new sprouted leaves and stems under white light (4.22 g) was 1.06 times of that under red light (3.98 g), 1.58 and 1.86 times of that under blue light $(2.67 \mathrm{~g})$ and green light $(2.27 \mathrm{~g})$ light, respectively. Therefore, the seedlings under white and red lights produced more biomass than that produced by the seedlings under blue and green lights.

Effects of different light quality on total gypenosides accumulation. The concentration of total gypenosides in the seedlings under red light $\left(25.20 \mu \mathrm{mol} \mathrm{g}^{-1} \mathrm{DW}\right)$ was similar to that under white light $\left(24.80 \mu \mathrm{mol} \mathrm{g}{ }^{-1} \mathrm{DW}\right)$ (Figure 1). The content of total gypenosides in the seedlings under blue light was $18.60 \mu \mathrm{mol} \mathrm{g}{ }^{-1} \mathrm{DW}$. Much less content of total gypenosides was found in the seedlings under green light $\left(5.75 \mu \mathrm{mol} \mathrm{g}{ }^{-1} \mathrm{DW}\right)$ (Figure 1). The content of total gypenosides in the seedlings under red light and white light were 4.38 and 4.31 times that under green light. It was also 1.35 and 1.33 times that under blue light. The content of total gypenosides under different lights seemed to be positively related to the dry weight $\left(r^{2}=0.8961\right)$.

Effects of different light quality on photosynthesis. Diurnal variation of Pn under different light quality.- The diurnal variation of net photosynthetic rate (Pn) presented a typical double-peak

Figure 1. Contents of total gypenosides in Gynostemma pentaphyllum seedlings grown under different light qualities.

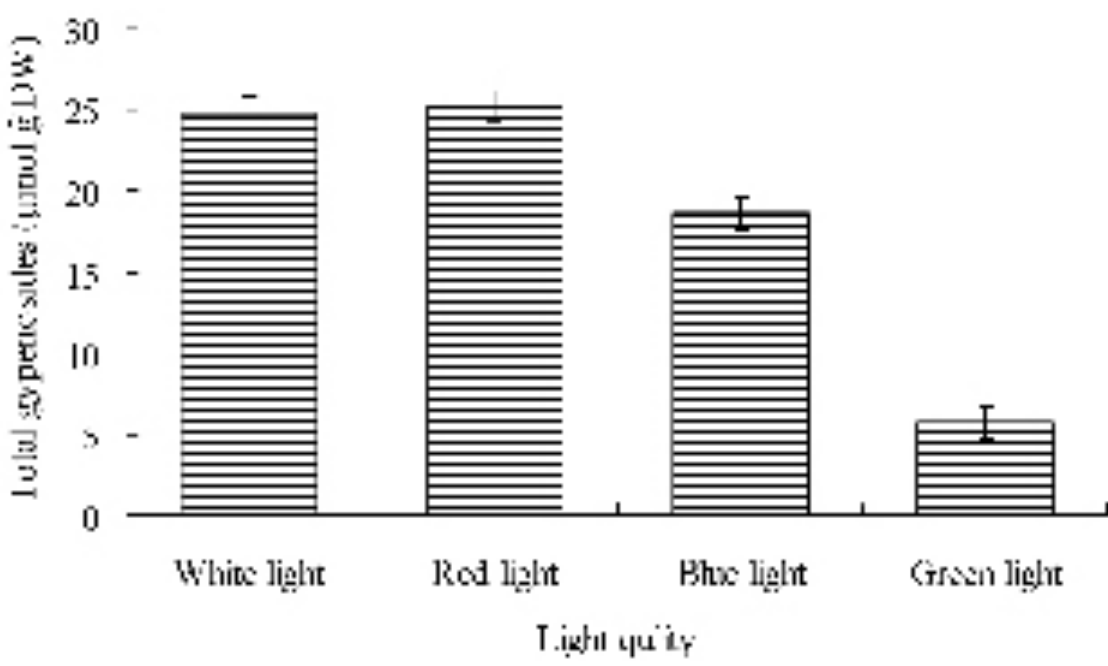




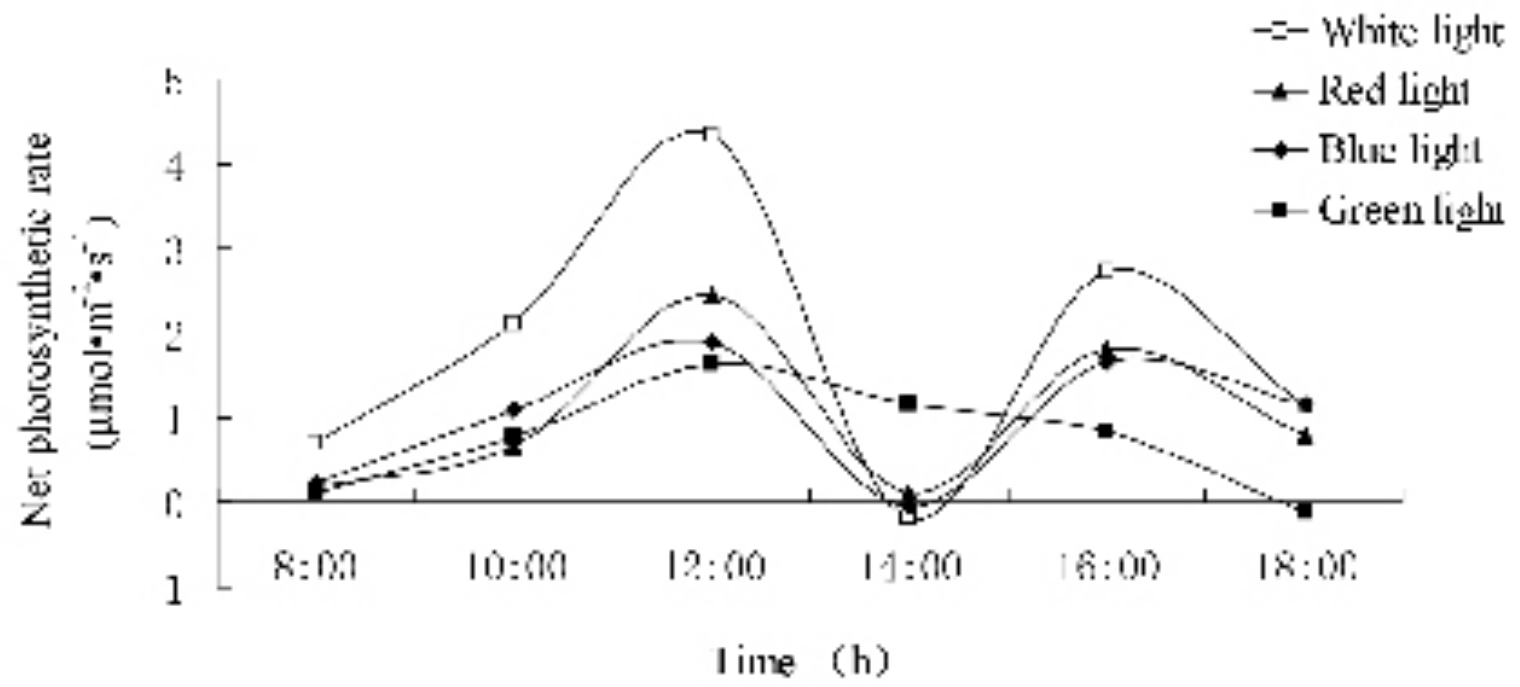

Figure 2. Diurnal variation of net photosynthetic rate of Gynostemma pentaphyllum seedlings grown under different light qualities.

curve when seedlings grown under white, red and blue lights, suggesting a remarkable phenomenon of "midday depression" occurred, while that under green light presented a single-peak curve (Figure 2). Although the diurnal variation pattern of Pn was simultaneously alike for seedlings under white and those under red and blue lights, the values of Pn were higher and changed sharply under white light. One of the two peaks in the double-peak curve appeared at 12:00 pm, the other appeared at 16:00 pm. The peak value at 12:00 pm was higher than the peak value at 16:00 pm. The dips in the double-peak curves under white, red and blue lights appeared at 14:00 and the Pn value was even negative under white and blue lights. By contrast, the diurnal variation of Pn presented a single-peak curve under green light and the peak occurred at 12:00 pm. Thereafter, the value slid down gradually and reached to the dip at 18:00 pm (Figure 2). The mean value of Pn under white light, red, blue and green lights was $1.802 \mu \mathrm{mol} \cdot \mathrm{m}^{-2} \cdot \mathrm{s}^{-1}, 1.005 \mu \mathrm{mol} \cdot \mathrm{m}^{-2} \cdot \mathrm{s}^{-1}$, $0.990 \mu \mathrm{mol} \cdot \mathrm{m}^{-2} \cdot \mathrm{s}^{-1}$ and $0.739 \mu \mathrm{mol} \cdot \mathrm{m}^{-2} \cdot \mathrm{s}^{-1}$, respectively. The order of mean value of Pn was basically in accordance with the order of biomass $\left(r^{2}=0.7702, P<0.05\right)$ and total gypenosides content $\left(r^{2}=0.6657\right.$ for 4 lights, $r^{2}=0.9583$ for monochromatic lights, $\left.P<0.05\right)$.

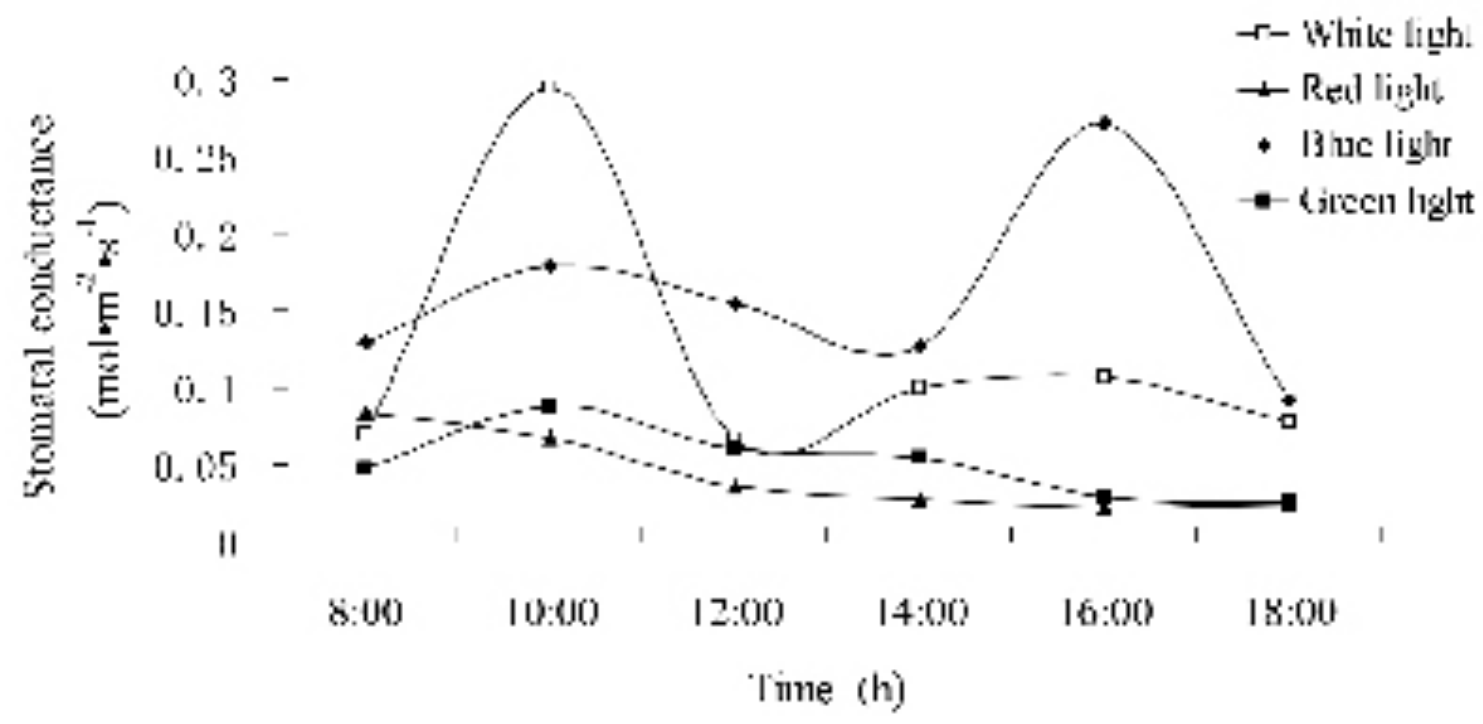

Figure 3. Diurnal variation of stomatal conductance of Gynostemma pentaphyllum seedlings grown under different light qualities. 
Figure 4. Diurnal variation of transpiration rate of Gynostemma pentaphyllum seedlings grown under different light qualities.
Figure 5. Diurnal variation of intercellular $\mathrm{CO}_{2}$ concentration of Gynostemma pentaphyllum seedlings grown under different light qualities.

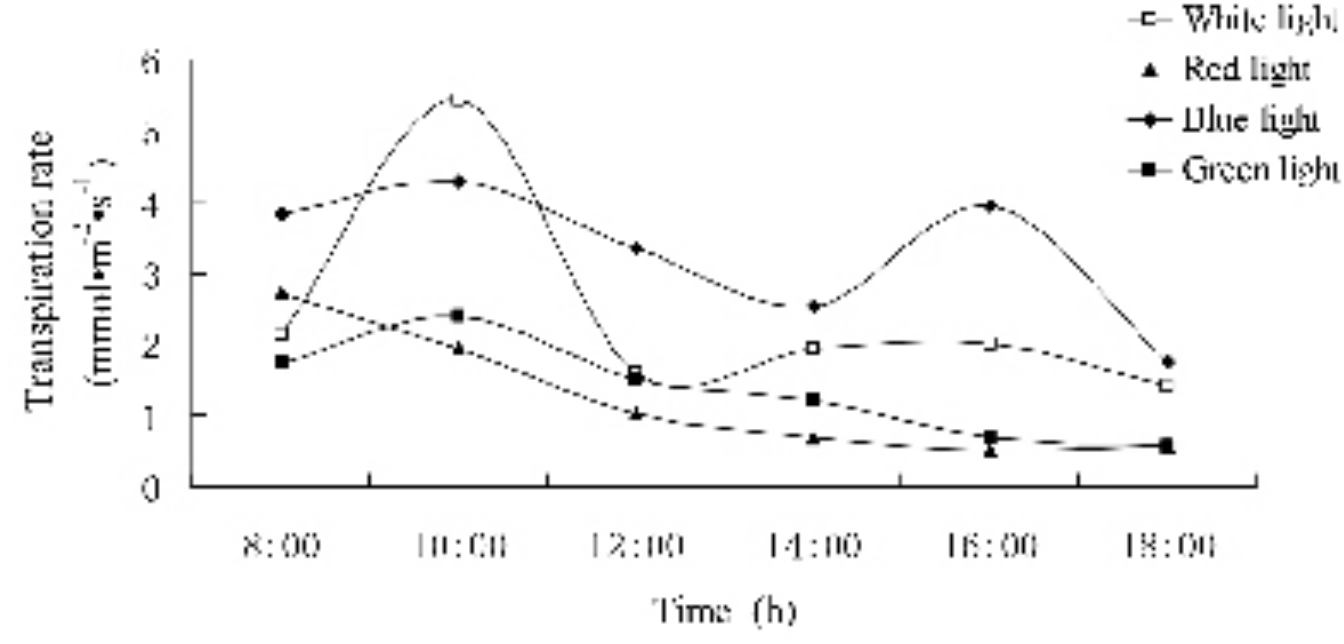

Diurnal variation of stomatal conductance under different light quality.- The diurnal variation curve of stomatal conductance (Cond) was a typical double-peak curve under white and blue lights, a single-peak curve under green light and a slide-down line under red light (Figure 3). The two peaks under white light appeared at 10:00 am and 16:00 pm with the former value higher than that of the latter. The dip in the double-peak curve occurred at 12:00 pm. Similarly, the two peaks under blue light were also appeared at 10:00 am and 16:00 pm. However, the former value was lower than the latter, and the time of dip was appeared at 14:00 pm. The curve of Cond under green light increased from 8:00 am to 10:00 am and arrived at the peak, then decreased until the end. The line under red light dropped gradually from the highest value at the beginning of 8:00 am to the ending of 18:00 pm (Figure 3).

Diurnal variation of transpiration rate under different light quality.- The pattern of diurnal variation curve of transpiration rate (T) (Figure 4) was closely paralleled to that of stomatal conductance. The two peaks under white light were at 10:00 am and 16:00 pm, with the dip appeared at 12:00 pm. Though the peak in the diurnal variation curve of $T$ under blue light presented at the same time as that under the white light, the dip under blue light appeared at 14:00 pm. Similarly, in the curve of both under white light and blue light, the value of the first peak was higher than that of the second peak. The only peak under green light appeared at 10:00 am and then declined gradually. The slide-down line under red light descended from the highest beginning at 8:00 am till the test ended. As shown in Figure 3 and Figure 4, the curve of stomatal conductance and the curve of transpiration rate fluctuated simultaneously. A significant positive correlation between $\mathrm{T}$ and Cond was observed under white, red and green light condition (white light, $r^{2}=0.9782$;

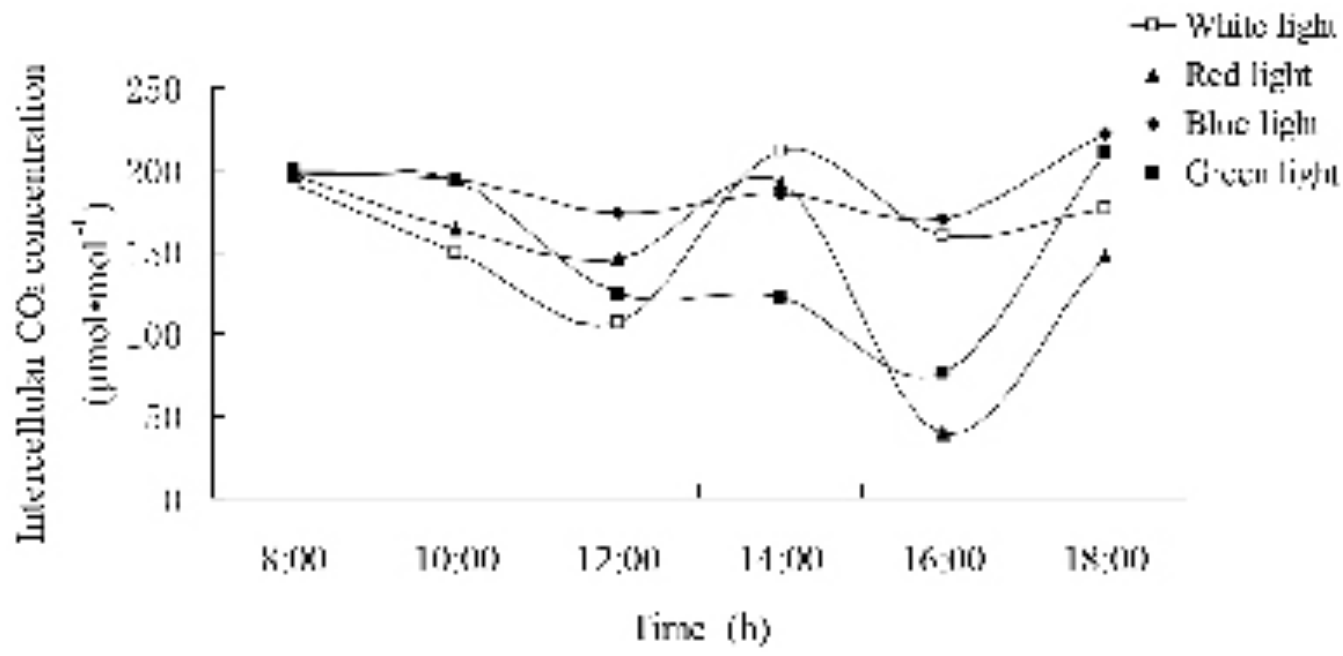


red light, $r^{2}=0.9961$ and green light, $r^{2}=0.9281, P<0.05$ ), while the positive correlation was non-significant under blue light $\left(r^{2}=0.6798\right)$. The statistical analysis indicated that there's no significant correlation between transpiration rate and $\mathrm{Pn}$, with $r^{2}=0.0313$ (white light), -0.4087 (red light), 0.1836 (blue light) and 0.2169 (green light), respectively.

Diurnal variation of intercellular $\mathrm{CO}_{2}$ concentration under different light quality.- The daily changes in intercellular $\mathrm{CO}_{2}(\mathrm{Ci})$ under white, red and blue lights presented a typical doubledip curve with one peak between them, while a single-dip curve was observed under green light (Figure 5). In the curves under white, red and blue lights, the two dips appeared at 12:00 pm and 16:00 pm, inserted by a peak that occurred at 14:00 pm. The value of the first dip was lower than the second under white light, whereas the changing trend under red light was reverse. The curve of $\mathrm{Ci}$ under blue light was not as typically as above, although the mean value of $\mathrm{Ci}$ was the highest among all lights. Single-dip curve of $\mathrm{Ci}$ was observed under green light, with the lowest value located at 16:00 pm and the highest value at 18:00 pm. Compared Figure 5 with Figure 2, it showed that the peaks and dips in the curve of $\mathrm{Ci}$ were basically opposite to that in curve of Pn under different lights, which suggested that Pn was negatively correlated with $\mathrm{Ci}$ in Gynostemma pentaphyllum, especially under white light $\left(r^{2}=-0.974, P<0.05\right)$.

\section{Discussion}

Light is one of the most important environmental factors in plant growth. Several works have demonstrated that the diurnal variation curve of photosynthetic rate of Gynostemma pentaphyllum is a typical double-peak curve with an obvious phenomenon of midday depression under natural light (Huang et al. 2004, Liu \& Hu 2004). However, little is known about the influence of light quality on the photosynthesis of G. pentaphyllum. The higher Pn under forest gaps than that under forest was ascribed to the intensities and spectra compositions of blue and red orange light (Wei et al. 2003). The present research revealed that the diurnal variation curves of Pn under white, red and blue lights were double-peak curves accompanied with midday depression, which were similar to those under full sunlight or shading conditions. By contrast, the Pn curve under green light had only one single-peak. The mean value of Pn was the highest under white light out of the monochromatic lights. Under the same irradiate intension, Pn under red light was the highest, and that under blue light was higher than that under green light, which was in accordance with the cases in such plants as wheat, lettuce and Ginkgo (Goins et al. 1997, Kim et al. 2004, Leng et al. 2002). In the present study, the daily change in the curve of Cond (Figure 3) and T (Figure 4) under different lights was coincided with the result of Gynostemma pentaphyllum under shade (Huang et al. 2004). We also showed that the curve of Ci (Figure 5) was opposite to the curve of Pn. Combining the fact that the midday depression and highest leaf temperature occurred at the same time, the above data suggested that the photosynthetic midday depression was not mainly associated with stoma, but with such non-stomatal factor as Rubisco in mesophyll cells. This suggestion is in accordance with the criterion of Farquhar \& Sharkey (1982).

Photosynthesis reflects the sustainable ability of plants to accumulate substances and undertake physiological metabolism. In the case of Hypericum perforatum L., the total fresh mass, dry mass and the number of stem nodes were greatest when the value of photosynthetic rate was the highest (Mosaleeyanon et al. 2005). By employed color film, Luo et al. (2014) revealed that red light increased the height of Panax notoginseng (Burkill) F. H. Chen ex C. Y. Wu \& K. M. Feng, while cyan, yellow, violet and blue lights promoted the accumulation of biomass underground, though the higher photosynthetic rate was under blue and yellow lights, and the green light had the least effect. Likely color film test demonstrated that the higher growth and biomass of plants were achieved under yellow film than green film, and red film performed better than blue film, respectively (Liu et al. 2011, Li et al. 2011). The present study showed that white light could significantly increase the growth and dry weight of Gynostemma pentaphyllum when compared with red, blue and green lights. This situation was exactly similar to that of cucumber seedlings (Su et al. 2014), for white light contains broader light spectrum than color lights do and thus has a higher efficient physiological impact. However, each monochromatic light played 
its special role in regulating the growth of G. pentaphyllum. Red light increased the biomass by enhancing the stem length and differentiation of leaves. Blue light promoted the elongation of petiole to seek more light sources. Green light decreased the biomass by inhibiting the length of stem and formation of new leaves. Generally, the effects of color lights on growth and biomass were closely related to the efficiency of photosynthesis in G. pentaphyllum.

The relationships between photosynthesis and secondary metabolite contents have attracted attention for decades in officinal plant utilization. Light quality affected the procedure of secondary metabolism of plants, and the intensity depended on species and growing conditions. For instance, the increase in the content of Hypericin and pseudohypericin in leaves of Hypericum perforatum accompanied with the increase in photosynthesis (Mosaleeyanon et al. 2005). Yan et al. (2003) reported that red film conducted an increase of $83 \%$ in salidroside content and of $35 \%$ in salidroside yield, whereas yellow, blue and green films did decreases of 56.95,72.22 and $31.94 \%$ in salidroside content and 70.43, 92.35 and $82.34 \%$ in salidroside yield, respectively. The results suggested that red film treatment enhanced the production and accumulation of salidroside in the roots of Rhodiola sachalinensis Boriss. According to Yu et al. (2005) the biomass of ginseng hairy roots cultivated in bioreactors was the highest under red light, followed by that under blue light and then the fluorescent light, while the content of ginsenoside was in opposite order. Fournier et al. (2003) presented red light (R), far red light (FR) and the R: FR ratio significantly affected the content of Rd, Rc, and $\mathrm{Rg}$ (1) ginsenosides in 2-year-old roots of American ginseng, with a variation up to $40 \%$. Panax notoginseng, an herb that contains effective components of notoginsenosides and ginsenosides, had the most accumulation of Rd ginsenoside under cyan light and total effective components under yellow and cyan lights (Luo et al. 2014). It was reported that red (4.96\% yield) and blue (4.75\% yield ) film treatments produced more total gypenosides in Gynostemma pentaphyllum than that upon yellow (1.36\% yield) and green (1.29\% yield) film treatments, while the highest yield was obtained upon full sunlight treatment (Liu et al.2011, Li et al.2011). The available data proved that the accumulation of ginsenosides and its analogues were closely associated with light quality. However, the relationship between the accumulation of secondary metabolites and photosynthetic efficiency remained unanswered. In this report, we confirmed that red light $(25.20 \mu \mathrm{mol} \mathrm{g-1DW})$ and white light $\left(24.80 \mu \mathrm{mol} \mathrm{g}^{-1}\right.$ DW) had an effect on enhancing the accumulation of total gypenosides in comparison with the effect of blue light $\left(18.60 \mu \mathrm{mol} \mathrm{g}{ }^{-1} \mathrm{DW}\right)$ and green light $\left(5.75 \mu \mathrm{mol} \mathrm{g}{ }^{-1} \mathrm{DW}\right)$. The gypenosides content under white light was approximately the same as that under red light, implied that the effect of white light on metabolites accumulation was involved in the effect of red light. This was basically in keeping with the results of Liu et al. (2011) and Li et al (2011). Moreover, a positive relationship could be found between the biomass, total gypenosides content and the net photosynthetic rate, as shown in Table 1, Figure 1 and 2, especially for monochromatic lights. It could be deduced that the effect of light quality on growth and accumulation of gypenosides depended upon the photosynthetic efficiency. That is, different light quality resulted in different photosynthetic efficiency, transformed different amounts of biomass and finally converted different amounts of secondary metabolites.

\section{Acknowledgments}

This work is supported by the National Natural Science Foundation of China (No.31260039) and the Key Course of Hunan Province (Ecology)/Jishou University. We are grateful to reviewers for their good comments and suggestions.

\section{Literature cited}

Blumert M, Liu JL. 1999. Jiaogulan China’s “Immortality” Herb. Badger. Torchlight Publishing Inc.

Chang CK, Chang KS, Lin YC, Liu SY, Chen CY. 2005. Hairy root culturues of Gynostemma pentaphyllum (Thunb.) Makino: a promising approach for the production of gypenosides as an alternative of ginseng saponins. Biotechnology Letters 27: 1165-1169. DOI 10.1007/s10529-005-8653-7

Darko E, Heydarizadeh P, Schoefs B, Sabzalian MR. 2014. Photosynthesis under artificial light: the shift in primary and secondary metabolism. Philosophical Transactions of the Royal Society B 369: 20130243. DOI: $10.1098 /$ rstb.2013.0243 
Received:

November 10th, 2015

Accepted:

March 15th, 2016
Deng M, Zhong S, Ren B, Ren B, Tu ZM. 2000. Studies on the effect of illumination intensity on the content of total saponin in Gynostemma pentaphylllum (Thunb.) Makino. Acta Academiael Hubei 21: 102-103.

Farquhar GD, Sharkey TD. 1982. Stomatal conductance and photosynthesis. Annual Review of Plant Physiology 33: 317-345. DOI: 10.1146/annurev.pp.33.060182.001533

Fournier AR, Proctor JTA, Gauthier L, Khanizadeh S, Bélanger A, Gosselin A, Dorais M. 2003. Understory light and root ginsenosides in forest-grown Panax quinquefolius. Phytochemistry 63: 777-782. DOI 10.1016/S0031-9422(03)00346-7

Goins GD, Yorio NC, Sanwo MM, Brown CS. 1997. Photomorphogenesis, photosynthesis, and seed yield of wheat plants grown under red light-emitting diodes (LEDs) with and without supplemental blue lighting. Journal of Experimental Botany 48: 1407-1413. DOI 10.1093/jxb/48.7.1407

Guo SZ, Zhang YS, Ma QK, Gao HJ, Wang JP. 2008. Anatomic characteristics and quality of Gynostemma pentaphyllum vegetative organs in different Niches. Chinese Journal of Tropical Crops 29: 472-477.

Huang C, Wu Z, Yao Y, Xu X. 2004. Photosynthetic characteristics of Gynostemma pentahyllum under shade. Journal of Applied Ecology 15 (11):2099-2103.

Huyen VTT, Phan DV, Thang P, Ky PT, Hoa NK, Ostenson CG. 2012. Antidiabetic effects of add-on Gynostemma pentaphyllum extract therapy with sulfonylureas in type 2 diabetic patients. Evidence-based Complementary and Alternative Medicine 2012: 1-7. DOI 10.1155/2012/452313

Im SA, Choi HS, Choi SO, Kim KH, Lee S, Hwang BY, Lee MK, Lee CK. 2012. Restoration of electric footshock-induced immunosuppression in mice by Gynostemma pentaphyllum components. Molecules 17, 7695-7708. DOI:10.3390/molecules 17077695

Kim HH, Goins GD, Wheeler RM, Sager JC. 2004. Green-light supplementation for enhanced lettuce growth under red- and blue-light-emitting diodes. HortScience 39: 1617-1622.

Leng PS, Su SC, Wang TH, Jiang XN, Wang SS. 2002. Effects of light intensity and light quality on photosynthesis, flavonol glycoside and terpene lactone contents of Ginkgo biloba L. seedlings. Journal of Plant Resources and Environment 11: 1-4.

Li XY, Liu SB, Tang KH, Yuan ZZ. 2011. Effects of color films on the growth and total gypenosides accumulation in Gynostemma pentaphyllum and Gynostemma pentagynum. Chinese Journal of Tropical Crops 32: 915-920.

Liu JY, Chen XX, Tang SC-W, Lao LX, Cho WSS., Lee KF, Zhang KYB. 2015. Edible plants from traditional Chinese medicine are a promising alternative for the management of diabetic nephropathy. Journal of Functional Foods 14:12-22. DOI 10.1016/j.jff.2015.01.034

Liu SB, Hu ZH. 2004. Effects of shading treatment on the leaf morphology, structure and photosynthetic characteristics of Gynostemma pentaphyllum. Journal of Wuhan Botanical Research 22: 339-344.

Liu SB, Li ZY, Tang KH, Li L, Li XY. 2011. Illumination factors affecting the growth, development and accumulation of total gypenosides in Gynostemma pentaphyllum and G.pentagynum. Chinese Journal of Tropical Crops 32: 50-54.

Luo MJ, Xia PG, Qi ZH, Zhang XH, Chen ZJ, Liu Y. 2014. Effect of light quality on growth, photosynthesis and effective components of Panax notoginseng. China Journal of Chinese Materia Medica 39: 610-613.

Lüthje P, Lokman EF, Sandström C, Östenson CG, Brauner A. 2015. Gynostemma pentaphyllum exhibits anti-inflammatory properties and modulates antimicrobial peptide expression in the urinary bladder. Journal of Functional Foods 17: 283-292. DOI 10.1016/j.jff.2015.03.028

Morrow RC. 2008. LED lighting in horticulture. Hortscience 43:1947-1950.

Mosaleeyanon K, Zobayed SMA, Afreen F, Kozai T. 2005. Relationships between net photosynthetic rate and secondary metabolite contents in St. John's wort. Plant Science 169: 523-531. DOI 10.1016/ j.plantsci.2005.05.002

Su N, Wu Q, Shen Z, Xia K, Cui J. 2014. Effects of light quality on the chloroplastic ultrastructure and photosynthetic characteristics of cucumber seedlings. Plant Growth Regulator 73: 227-235. DOI: 10.1007/s 10725-013-9883-7

Wei C, Sun Q, Peng Z, Yan D. 2003. Light environment characteristics of forest gap in deciduous broadleaved forest and its effects on growth features of Gynostemma pentaphyllum in Jianghuai watershed. The Journal of Applied Ecology 14: 665-670.

Yan H, Wang X, Niu J, Wang Y, Wang P, Liu Q. 2014. Anti-cancer effect and the underlying mechanisms of gypenosides on human colorectal cancer SW-480 cells. PLOS ONE 9 (4): e95609. doi:10.1371/journal. pone.0095609

Yan X, Wang Y, Shang X. 2003. Effects of greenhouse light intensity and quality on biomass and salidroside content in roots of Rhodiola sachalinensis. Acta Ecologica Sinica 23: 841-849.

Yu KW, Murthy HN, Hahn EJ, Paek KY. 2005. Ginsenoside production by hairy root cultures of Panax ginseng: influence of temperature and light quality. Biochemical Engineering Journal 23: 53-56. DOI 10.1016/j.bej.2004.07.001 